\title{
Polariton dynamics and Bose-Einstein condensation in semiconductor microcavities
}

\author{
D. Porras, ${ }^{1}$ C. Ciuti, ${ }^{2}$ J. J. Baumberg, ${ }^{3}$ and C. Tejedor ${ }^{1}$ \\ ${ }^{1}$ Departamento de Física Teórica de la Materia Condensada, Universidad Autónoma de Madrid, 28049 Cantoblanco, Madrid, Spain \\ ${ }^{2}$ Physics Department, University of California San Diego, 3500 Gilman Drive, La Jolla, California 92093 \\ ${ }^{3}$ Department of Physics \& Astronomy, University of Southampton, Southhampton SO17 IBJ, United Kingdom
}

(Received 12 February 2002; revised manuscript received 20 May 2002; published 5 August 2002)

\begin{abstract}
We present a theoretical model that allows us to describe the polariton dynamics in a semiconductor microcavity at large densities, for the case of nonresonant excitation. Exciton-polariton scattering from a thermalized exciton reservoir is identified as the main mechanism for relaxation into the lower polariton states. A maximum in the polariton distribution that shifts towards lower energies with increasing pump power or temperature is shown, in agreement with recent experiments. Above a critical pump power, macroscopic occupancies $\left(5 \times 10^{4}\right)$ can be achieved in the lowest-energy polariton state. Our model predicts the possibility of Bose-Einstein condensation of polaritons, driven by exciton-polariton interaction, at densities well below the saturation density for CdTe microcavities.
\end{abstract}

DOI: 10.1103/PhysRevB.66.085304

PACS number(s): 71.36.+c, 42.55.Sa, 71.35.Lk, 03.75.Fi

\section{INTRODUCTION}

Polaritons are quasiparticles created by the strong coupling between excitons and photons, and behave as composite bosons at small enough densities. In the last decade a huge experimental and theoretical effort has been dedicated to the search for quantum degeneracy effects in microcavity polaritons. They were soon proposed as candidates for the formation of a Bose-Einstein condensate, due to their small density of states (DOS). ${ }^{1}$ However, a bottleneck effect due to the slow polariton-acoustical phonon scattering was predicted $^{2}$ and observed in angle-resolved experiments. ${ }^{3}$ Stimulated scattering due to the bosonic nature of polaritons has been demonstrated ${ }^{4}$ in pump-probe experiments. Parametric amplification and parametric oscillation, under resonant excitation, has been observed ${ }^{5,6}$ and was well explained by coherent polariton-polariton scattering. ${ }^{7-9}$ In GaAs microcavities, experiments have shown the role of polaritonpolariton scattering in the nonlinear emission after nonresonant excitation, either in continous-wave, ${ }^{10-12}$ or timeresolved experiments. ${ }^{13}$ The growth of II-VI microcavities leads to exciting possibilities due to the larger stability of the exciton. The study of these samples has allowed for the observation of stimulated scattering in the strong-coupling regime under nonresonant excitation conditions. ${ }^{14}$ The robustness of the polariton in these samples has been recently shown by the demonstration of parametric amplification at high temperatures. ${ }^{15}$

The theoretical study of semiconductor microcavities has followed two different lines. A fermionic formalism, including carrier-carrier correlation, explained the lasing by population inversion at densities above the saturation density, where the coupling between the exciton and the photon disappears (weak-coupling regime). ${ }^{16,17}$ On the other hand, a bosonic picture, including polariton-polariton interaction, has been developed. It must be stressed that recent experiments agree with the predictions made by the bosonic formalism (bottleneck effect, stimulated scattering) under excitation conditions such that the density of polaritons remains below the saturation density. The polariton-polariton interaction, given by the usual Coulomb exchange interaction between excitons and the saturation term, has also explained quantitatively many recent observations. ${ }^{4-8}$

In this paper we present a theoretical study of the evolution of the polariton population at large densities with a bosonic description. We use the semiclassical Boltzmann equation, developed by Tassone and Yamamoto, ${ }^{18}$ as the starting point for the description of the polariton dynamics. Exciton-polariton $(X-P)$ scattering is identified as the most important mechanism for relaxation towards the lower polariton branch. We deduce a simplified model in which the high-energy excitons are considered as a thermalized reservoir. This simplification allows us to describe all the microscopic lower polariton states, and the evolution of the polariton population towards Bose-Einstein condensation (BEC). ${ }^{19}$

$X-P$ scattering is shown to produce a polariton population with an occupancy that has a maximum at a given energy below the exciton energy. The energy at which the polariton distribution peaks is shown to decrease linearly as pump power or sample temperature is increased, in such a way that the dip in the polariton dispersion behaves as a polariton trap in phase space. ${ }^{20}$ This conclusion is valid for all semiconductor microcavities. In particular, the same behavior has been observed in a recent experiment in a GaAs microcavity. ${ }^{10}$ However, in GaAs, densities comparable to the saturation density are created, as one increases the pump power, before the threshold for BEC is reached. Thus, for a clean observation of all the phenomenology described in this paper, better material parameters than those of GaAs microcavities are desirable. We predict that in CdTe microcavities, where excitons are more stable due to their smaller Bohr radius, the threshold for BEC can be reached for densities well below the saturation density. Thus, we choose, for concreteness, CdTe parameters for the detailed calculations shown below. Our model shows that the relaxation towards the ground state is only possible for densities comparable to the saturation density in GaAs microcavities. In order to achieve BEC with smaller polariton densities, a scattering channel, addition to those considered here should be included. Electron-polariton 
scattering in doped microcavities has been recently proposed $^{21}$ as an efficient mechanism that allows BEC for such small polariton densities.

The paper is organized as follows. In Sec. II we present the theoretical framework. A set of differential equations is obtained for the description of the microscopic lower polariton levels, and the exciton density and exciton temperature. In Sec. III, we apply this model to the study of the dynamics of the polariton population for different pump powers and temperatures, after nonresonant, continuous excitation. The case of Bose-Einstein condensation into the lowest-energy polariton mode is considered in Sec. IV. Finally, in Sec. V, we list our main conclusions and discuss the comparison with experiments.

\section{RATE EQUATION SIMPLIFIED MODEL}

\section{A. Simplified model for the exciton-polariton scattering}

At densities such that $n a_{B}^{2} \ll 1$, where $a_{B}$ is the twodimensional (2D) exciton Bohr radius, excitons can be described as interacting bosons. From typical parameters for CdTe QW's (exciton binding energy $E_{B} \approx 25 \mathrm{meV}$, and electron and hole effective masses $m_{e}=0.096, m_{h}=0.2$ ), we get a 2D exciton Bohr radius $a_{B}=47 \AA$ and $a_{B}^{-2}=4.5$ $\times 10^{12} \mathrm{~cm}^{-2}$. On the other hand, at large exciton densities, carrier exchange interactions and phase-space filling effects destroy the coupling between excitons and photons. The density at which the exciton oscillator strength is completely screened is the saturation density $n_{\text {sat }}$. Following SchmittRink et al., ${ }^{22}$ we can estimate for a CdTe microcavity,

$$
n_{\text {sat }}=\frac{0.117}{\pi a_{\text {eff }}^{2}}=6.7 \times 10^{11} \mathrm{~cm}^{-2},
$$

where $a_{\text {eff }}^{2}=a_{B} / 2$. In InGaAs and GaAs QW's, $n_{\text {sat }}$ would be $6.6 \times 10^{10} \mathrm{~cm}^{-2}$ and $1.3 \times 10^{11} \mathrm{~cm}^{-2}$, respectively. Throughout this paper we consider the case of CdTe in a range of polariton densities well below $n_{\text {sat }}$, so that the microcavity remains in the strong-coupling regime.

In the strong-coupling regime, the Hamiltonian includes the coupling between excitons and photons through the polariton splitting, $\Omega_{P}:{ }^{23}$

$$
H_{0}=\sum_{\mathbf{k}}\left[\epsilon_{\mathbf{k}}^{x} b_{\mathbf{k}}^{\dagger} b_{\mathbf{k}}+\epsilon_{\mathbf{k}}^{c} a_{\mathbf{k}}^{\dagger} a_{\mathbf{k}}+\frac{\Omega_{P}}{2}\left(a_{\mathbf{k}}^{\dagger} b_{\mathbf{k}}+b_{\mathbf{k}}^{\dagger} a_{\mathbf{k}}\right)\right] .
$$

$b_{\mathbf{k}}^{\dagger}, \epsilon_{\mathbf{k}}^{x}\left(a_{\mathbf{k}}^{\dagger}, \epsilon_{\mathbf{k}}^{c}\right)$ are the creation operator and dispersion relation for the bare exciton (photon) mode. The residual Coulomb interaction between excitons and the saturation of the exciton oscillator strength can be described by the effective exciton-exciton and exciton-photon interaction Hamiltonian: ${ }^{24}$

$$
\begin{aligned}
H_{I}= & \sum_{\substack{\mathbf{k}_{1}, \mathbf{k}_{2} \\
\mathbf{q}}} \frac{M_{x x}}{2 S} b_{\mathbf{k}_{1}}^{\dagger} b_{\mathbf{k}_{2}}^{\dagger} b_{\mathbf{k}_{1}+\mathbf{q}} b_{\mathbf{k}_{2}-\mathbf{q}} \\
& +\frac{\sigma_{\text {sat }}}{S} b_{\mathbf{k}_{1}}^{\dagger} b_{\mathbf{k}_{2}}^{\dagger} b_{\mathbf{k}_{1}+\mathbf{q}} a_{\mathbf{k}_{2}-\mathbf{q}}+\text { H.c. }
\end{aligned}
$$

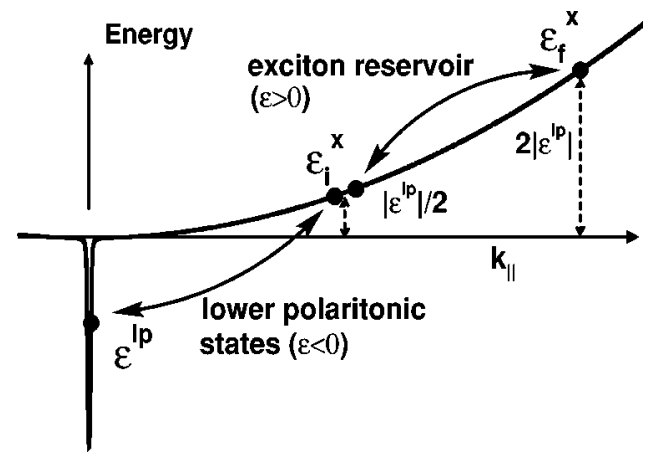

FIG. 1. Exciton-polariton scattering process from a thermalized exciton reservoir into the lower polariton branch.

where $S$ represents the quantization area. We consider here the small-momentum limit for $H_{I}$, an approximation that is valid for the range of small wave vectors considered below. The matrix elements in $H_{I}$ are given by

$$
\begin{gathered}
M_{x x} \approx 6 E_{B} a_{B}^{2}, \\
\sigma_{\text {sat }} \approx 1.8 \Omega_{P} a_{B}^{2} .
\end{gathered}
$$

$H_{0}$ can be diagonalized in the polariton basis:

$$
\begin{gathered}
p_{l p, \mathbf{k}}=X_{\mathbf{k}}^{l p} b_{\mathbf{k}}+C_{\mathbf{k}}^{l p} a_{\mathbf{k}}, \\
p_{u p, \mathbf{k}}=X_{\mathbf{k}}^{u p} b_{\mathbf{k}}+C_{\mathbf{k}}^{u p} a_{\mathbf{k}},
\end{gathered}
$$

where $X_{\mathbf{k}}^{l p / u p}, C_{\mathbf{k}}^{l p / u p}$ are the Hopfield coefficients that represent the excitonic and photonic weights in the polariton wave function for the lower/upper branch, respectively. ${ }^{25} \mathrm{By}$ means of the transformation (5) we can express $H_{I}$ in terms of polariton operators. We will neglect the upper polariton branch, an approximation that will be justified later. We express the operators $b_{\mathbf{k}}, a_{\mathbf{k}}$ in Eq. (3) in terms of $p_{l p, \mathbf{k}}, p_{u p, \mathbf{k}}$ and retain the terms corresponding to interactions within the lower polariton branch only,

$$
\begin{aligned}
H= & H_{0}+H_{I}=\sum_{\mathbf{k}} \epsilon_{\mathbf{k}}^{l p} p_{l p, \mathbf{k}}^{\dagger} p_{l p, \mathbf{k}} \\
& +\sum_{\substack{\mathbf{k}_{1}, \mathbf{k}_{2} \\
\mathbf{k}_{3}, \mathbf{k}_{4}}} \frac{1}{2 S} V_{\mathbf{k}_{1}, \mathbf{k}_{2}, \mathbf{k}_{3}, \mathbf{k}_{4}}^{l p-l p} p_{l p, \mathbf{k}_{1}}^{\dagger} p_{l p, \mathbf{k}_{2}}^{\dagger} p_{l p, \mathbf{k}_{3}} p_{l p, \mathbf{k}_{4}},
\end{aligned}
$$

where

$$
\begin{aligned}
\frac{1}{2} V_{\mathbf{k}_{1}, \mathbf{k}_{2}, \mathbf{k}_{3}, \mathbf{k}_{4}}^{l p-l p}= & \left(\frac{1}{2} M_{x x} X_{\mathbf{k}_{1}}^{l p} X_{\mathbf{k}_{2}}^{l p} X_{\mathbf{k}_{3}}^{l p} X_{\mathbf{k}_{4}}^{l p}+\sigma_{s a t} C_{\mathbf{k}_{1}}^{l p} X_{\mathbf{k}_{2}}^{l p} X_{\mathbf{k}_{3}}^{l p} X_{\mathbf{k}_{4}}^{l p}\right. \\
& \left.+\sigma_{s a t} X_{\mathbf{k}_{1}}^{l p} X_{\mathbf{k}_{1}}^{l p} C_{\mathbf{k}_{3}}^{l p} X_{\mathbf{k}_{4}}^{l p}\right) \delta_{\mathbf{k}_{1}+\mathbf{k}_{2}, \mathbf{k}_{3}+\mathbf{k}_{4}}
\end{aligned}
$$

$\epsilon_{\mathbf{k}}^{l p}$ is the dispersion relation for the lower polariton branch. It is plotted, assuming typical parameters for a semiconductor microcavity, in Fig. 1. For energies higher than the bare exciton energy, it matches the bare exciton dispersion.

The theoretical model developed by Tassone and Yamamoto ${ }^{18}$ describes the evolution of the polariton popula- 
tion by means of a semiclassical Boltzmann equation that includes polariton-acoustical-phonon and polaritonpolariton scattering. A grid uniform in energy is considered for the description of the polaritonic levels. Here, we are interested in the evolution of the system towards a BoseEinstein condensate. It is well known that in 2D systems, $\mathrm{BEC}$ is possible at finite temperatures only for systems with a finite quantization length $L_{c}$. As a result, the critical density and temperature depend logarithmically on this length scale. Due to the peculiarity introduced by the $2 \mathrm{D}$ character of semiconductor microcavities, the grid uniform in energy considered in Ref. 18 is not well suited for the study of the transition towards BEC. Instead, a uniform grid in $k$ space, related with the inverse of the quantization length, $L_{c}^{-1}$, has to be considered. Unfortunately, the number of levels that one has to consider for the description of the polariton dynamics is dramatically increased, and a numerical calculation that includes all the possible polariton-polariton scattering processes is not possible. Thus some simplification has to be done, which still allows to describe satisfactorily the polariton dynamics, and to predict the possibility of Bose-Einstein condensation.

In order to develop a simplified model for the case of nonresonant excitation, we make the following assumptions.

(1) The exciton population above the bare exciton energy can be considered as a reservoir at a given temperature, which follows a Maxwell-Boltzmann distribution.

(2) The main scattering mechanism at large densities for relaxation into the lower polariton modes is the one shown in Fig. 1, in which two excitons scatter in the reservoir, and the final states are a lower polariton and another reservoir exciton. We will call this process exciton-polariton scattering.

A scattering process in which two excitons are initially in the exciton reservoir, and a lower and upper polariton are the final states is also possible, but has a much smaller probability. This is due to the fact that in such a collision, an upper polariton, instead of an exciton, plays the role of a final state. Thus, the scattering rate is reduced by the ratio between the upper polariton and bare exciton DOS, $\rho_{u p} / \rho_{x} \approx 4 \times 10^{-5}$. In fact, for negative or zero detunings, the upper polariton population remains negligible, as shown experimentally ${ }^{14}$ and theoretically. ${ }^{18}$ The scattering of excitons with acoustical phonons going into lower polariton states is also much slower than the $X-P$ scattering already at exciton densities of $10^{9} \mathrm{~cm}^{-2}$ and the lattice temperatures considered here.

The phase space is, thus, divided into two parts.

(1) The exciton reservoir, which describes the excitons directly created by the nonresonant pump. We take as zero energy the bare exciton energy, so that the exciton reservoir includes all the levels with $\epsilon>0$. These levels are weakly coupled to the photonic modes, so that they can be approximated as bare excitons. The occupation numbers in the exciton reservoir are

$$
N_{i}^{x}=N^{x} e^{-\epsilon_{i}^{x} / k_{B} T_{x}}
$$

$N^{x}$ is given by

$$
N^{x}=\frac{2 \pi n_{x}}{\rho_{x} k_{B} T_{x}},
$$

where $n_{x}$ is the exciton density, $\rho_{x}$ is the bare exciton density of states,

$$
\rho_{x}=\left(m_{e}+m_{h}\right) / \hbar^{2},
$$

and $T_{x}$ is the temperature of the exciton reservoir. The assumption of a rigid thermal exciton distribution is well justified, as discussed below, for levels above $\epsilon=0$, due to the fast exciton-exciton scattering that thermalizes the exciton population at large densities. ${ }^{18,26}$ On the other hand, a Maxwell-Boltzmann distribution, rather than a Bose-Einstein distribution, can be used to describe the exciton reservoir, provided that $N^{x}<<1 . N^{x} \approx 0.2$ for the largest densities considered here, so that quantum degeneracy effects are negligible. The main mechanism for radiative losses in the exciton reservoir is due to the coupling to the leaky modes, which we include by considering an exciton lifetime $\tau_{x}=100 \mathrm{ps}^{27}$

(2) The lower polariton branch $(L P B)$, where the energy levels are below the bare exciton energy $\left(\epsilon_{k}^{l p}<0\right)$. Exciton and photon levels in the LPB are strongly coupled and radiative losses are much more important, due to the photonic weight of these states. Thus, we expect a nonequilibrium polariton distribution that depends strongly on temperature and density. On the other hand, the abrupt dispersion relation implies a very small effective mass for the LPB states, $m_{l p}$ $\approx\left(4 \times 10^{-5}\right) m_{x}$, leading to the possibility of Bose-Einstein condensation.

With this separation of the phase space we are neglecting the details of the polariton population near $\epsilon \approx 0$, that is, at the knee in the polariton dispersion relation, where the LPB and the quadratic dispersion of the bare excitons merge together (see Fig. 1). The contribution from these states is negligible already at the lowest temperatures considered here ( $4 \mathrm{~K}, k_{B} T \approx 0.35 \mathrm{meV}$ ), because the main part of the exciton distribution is in the weak coupling region.

We describe the LPB states by a grid in $k$ space. The exact quantization area is not well determined in experiments, due to inhomogeneities in the spot and surface defects. The relevant quantity is the quantization length that gives the order of magnitude for the distance between the energy levels. We consider first $L_{c}=50 \mu \mathrm{m}$ (a typical excitation spot diameter) and plane waves for the microscopic polariton states, so that the quantization area is simply $S=L_{c}^{2}$. The allowed wave vectors are given by

$$
\mathbf{k}^{l p}=\left(n_{\mathbf{x}} \mathbf{x}+n_{\mathbf{y}} \mathbf{y}\right) \frac{2 \pi}{L_{c}},
$$

where $n_{\mathbf{x}}, n_{\mathbf{y}}$ are integers. A similar method has been employed to describe the microscopic polariton levels in a microcavity etched into a microscopic post structure $\left(L_{c}\right.$ $=2 \mu \mathrm{m}){ }^{28}$ In that case, the LPB is reduced to a single microscopic level. On the contrary, in our case, $L_{c}$ is larger, and the number of lower polariton levels is of the order of $10^{3}$. The main results of our work do not depend strongly on the exact value of $L_{c}$, but only on its order of magnitude, as we show in the last section. The weak dependence of our 
results on $L_{c}$ can be easily understood from the peculiarities of BEC in 2D (see Appendix B).

We write now a set of rate equations for $N_{k}^{l p}$ (occupation numbers in the lower polariton branch) and $n_{x}$ (the density of the thermalized exciton reservoir). We consider the injection of polaritons from isotropically distributed excitons, so that the occupation numbers $N_{k}^{l p}$ will depend on the absolute value of the in-plane wave vector only. The rates for scattering from the thermalized reservoir into and out of the LPB due to the interaction Hamiltonian (3) are calculated in Appendix A. They lead to a simplified version of the semiclassical Boltzmann equation:

$$
\begin{aligned}
\frac{d N_{k}^{l p}}{d t}= & W_{k}^{\text {in }} n_{x}^{2}\left(1+N_{k}^{l p}\right)-W_{k}^{\text {out }} n_{x} N_{k}^{l p}-\Gamma_{k}^{l p} N_{k}^{l p} \\
\frac{d n_{x}}{d t}= & -\frac{1}{S} \sum_{k} d g_{k}^{l p}\left[W_{k}^{\text {in }} n_{x}^{2}\left(1+N_{k}^{l p}\right)-W_{k}^{\text {out }} n_{x} N_{k}^{l p}\right] \\
& -\Gamma_{x} n_{x}+p_{x} .
\end{aligned}
$$

$\Gamma_{k}^{l p}$ and $\Gamma_{x}$ are the radiative losses in the LPB and exciton reservoir, respectively:

$$
\begin{gathered}
\Gamma_{k}^{l p}=\left|C_{k}^{l p}\right|^{2} \frac{1}{\tau_{c}}, \\
\Gamma_{x}=\frac{1}{\tau_{x}},
\end{gathered}
$$

where $\tau_{c}$ is the lifetime for the bare photon within the cavity. We consider $\tau_{c}=1 \mathrm{ps}$, a typical value for high quality microcavities. $p_{x}$ represents the nonresonant pump that injects excitons directly into the exciton reservoir. If relaxation into the LPB is slow enough, the lifetime for an exciton in the exciton reservoir is given by $\tau_{x}$, so that a given pump $p_{x}$ $=n_{x} / \tau_{x}=n_{x} / 100 \mathrm{ps}$ creates an exciton density $n_{x}$. If scattering into the LPB is fast enough, however, the density created by the pump is smaller, as we will see below. $d g_{k}^{l p}$ is the degeneracy for the polariton level corresponding to the wave vector $k$, and is calculated according to the distribution obtained from Eq. (11).

$W_{k}^{\text {in }} n_{x}^{2}$ and $W_{k}^{\text {out }} n_{x}$ are the rates for $X-P$ scattering into and out of the LPB. They are given by the expressions (see Appendix A)

$$
\begin{aligned}
& W_{k}^{i n}=\frac{2 \pi}{\hbar k_{B} T_{x}} M_{k}^{2} e^{\epsilon_{k}^{l p} / k_{B} T_{x}}, \\
& W_{k}^{\text {out }}=\frac{1}{\hbar} M_{k}^{2} \rho_{x} e^{2 \epsilon_{k}^{l p} / k_{B} T_{x}},
\end{aligned}
$$

where

$$
M_{k}=M_{x x} X_{k}^{l p}+\sigma_{s a t} C_{k}^{l p} .
$$

The dependence of the rates on temperature can be qualitatively understood by the phase-space restrictions for relaxation into and out of the LPB (Fig. 1). Let us consider two excitons in the exciton reservoir with a wave vector $k_{i}^{x}$ and energy $\epsilon_{i}^{x}$, which scatter to a LPB state with energy $\epsilon_{k}^{l p}$ and to a high-energy state with $k_{f}^{x}$, $\epsilon_{f}^{x}$. Taking into account that the wave vector of the low-energy polariton is negligible, when compared with the wave vectors in the reservoir, simultaneous energy and momentum conservation implies that

$$
\begin{gathered}
2\left|k_{i}^{x}\right|=\left|k_{f}^{x}\right| \rightarrow 4 \epsilon_{i}^{x}=\epsilon_{f}^{x}, \\
2 \epsilon_{i}^{x}=-\left|\epsilon_{k}^{l p}\right|+\epsilon_{f}^{x} \rightarrow \epsilon_{i}^{x}=\left|\epsilon_{k}^{l p}\right| / 2, \epsilon_{f}^{x}=2\left|\epsilon_{k}^{l p}\right| .
\end{gathered}
$$

Equation (16) means that two excitons need an energy $\left|\epsilon_{k}^{l p}\right| / 2$ to be the initial states of a process in which one of them falls into the LPB. On the other hand, an exciton needs an energy $2\left|\epsilon_{k}^{l p}\right|$ in order to scatter with a lower polariton and take it out of the LPB.

The polariton distribution in the steady-state regime will be given by the set of equations (12) with $d N_{k}^{l p} / d t=0$, $d n_{x} / d t=0$. In the steady state, the occupation numbers in the LPB are given by

$$
N_{k}^{l p}=\frac{W_{k}^{i n} n_{x}^{2}}{W_{k}^{\text {out }} n_{x}+\Gamma_{k}^{l p}-W_{k}^{i n} n_{x}^{2}} .
$$

If we neglect the dependences on $k$ of the matrix element $M_{k}$ and the radiative losses $\Gamma_{k}^{l p}$, then the maximum in the polariton distribution has a peak at the energy

$$
\left|\epsilon_{\max }^{l p}\right|=\frac{1}{2} k_{B} T_{x} \ln \left(\frac{1}{\hbar \Gamma^{l p}} M^{2} \rho_{x} n_{x}\right) .
$$

Equation (18) implies an approximately linear relation between the exciton reservoir temperature and the energy position at which the system prefers to relax into the LPB (that is, the maximum energy loss in the relaxation process).

\section{B. Model for the evolution of the exciton reservoir temperature}

The strong dependence of the $X-P$ scattering into the LPB on $T_{x}$ implies that, in order to complete our model, we have to describe properly the evolution of the exciton reservoir temperature. During the relaxation process into the LPB, shown in Fig. 1, a high-energy exciton is injected into the exciton reservoir. This way, during the relaxation process, the exciton reservoir is heated. On the other hand, the scattering with acoustical phonons tries to keep the exciton reservoir at the lattice temperature. In order to describe the evolution of the exciton reservoir temperature, a new variable has to be added to this set of equations. We introduce $e_{x}$, the density of energy for the exciton reservoir, which is given for a Maxwell-Boltzmann distribution by the expression

$$
e_{x}=\frac{1}{S} \sum_{k} N_{k}^{x} \epsilon_{k}^{x}=n_{x}\left(k_{B} T_{x}\right) .
$$

The evolution of the energy allows us to obtain the exciton temperature at each step in the evolution of the polariton population.

However, we still need to make an assumption about the exciton distribution that the pump directly injects into the 
reservoir. Nonresonant experiments usually create a population of high-energy electron-hole pairs that relax towards low-energy states after the process of exciton formation.,29 It must be stressed that for the case of CdTe QW's this process is particularly fast, due to the possibility of exciton formation through the emission of LO phonons whose energy is $21 \mathrm{meV}$ below the exciton binding energy $(25 \mathrm{meV})$. The time scale for thermalization due to exciton-exciton scattering is very short $(\approx 1 \mathrm{ps})$, as compared with the time scale for relaxation into the LPB $(\approx 20 \mathrm{ps}$, for the larger exciton densities considered here). Thus, it is very well justified to describe the nonresonant pump as a source that injects thermalized excitons at a given pump temperature into the exciton reservoir. We still need to specify which is the initial pump temperature. Due to the large uncertainties in nonresonant experiments it is very difficult to make predictive calculations about this initial condition. We choose instead to make the simplest assumption: we consider that the pump injects excitons at the lattice temperature, $T_{L}$. For large exciton densities (above $5 \times 10^{10} \mathrm{~cm}^{-2}$ ) the processes of reservoir heating by $X-P$ scattering and cooling through exciton-phonon scattering govern the final exciton temperature, and our results do not depend on our assumption for the pump temperature. For small temperatures (below $10 \mathrm{~K}$ ) and densities, our results depend on this assumption. In a real experiment, the exciton temperature can be larger than that considered here, if the cooling of the initial nonresonantly created excitons is not fast enough.

It must be pointed out that a very efficient experimental setup for nonresonant excitation of a cold exciton distribution in a semiconductor microcavity has been demonstrated by Savvidis et al. ${ }^{10}$ In this experiment, nonresonant excitation has been achieved with small excess energies (below the stop band) due to the finite transmittance of the Bragg reflectors. The experimental results presented in (Ref. 10) are in agreement with a model in which excitons relax from a thermalized distribution into the lowest-energy states and justify all the assumptions made above.

We have to quantify the three contributions for the evolution of the exciton temperature listed above:

1. Heating by the scattering to high-energy exciton levels

The total energy is conserved in the $X-P$ scattering towards the LPB. Thus, the energy lost by the polaritons falling into the lower-energy states must be gained by the exciton reservoir. The following expression gives the rate of change of $e_{x}$ by each $X-P$ scattering process:

$$
\left.\frac{d e_{x}}{d t}\right|_{X-P}=-\frac{1}{S} \sum_{k} \epsilon_{k}^{l p} d g_{k}^{l p}\left[W_{k}^{i n} n_{x}^{2}\left(1+N_{k}^{l p}\right)-W_{k}^{\text {out }} n_{x} N_{k}^{l p}\right] .
$$

The heating of the exciton gas in the relaxation towards the LPB was also shown theoretically in Ref. 28 for the case of a microcavity post.

\section{Cooling produced by acoustical phonons}

The exciton-phonon scattering tries to keep excitons at the lattice temperature $T_{L}$ against the heating considered above. ${ }^{26}$ We divide the exciton Maxwell-Boltzmann distribution into levels equally spaced in energy with a given $\Delta E$ $\ll k_{B} T_{L}, k_{B} T_{x}$. The probability for absorption or emission of acoustical phonons gives a scattering rate $W_{j \rightarrow i}^{p h}\left(T_{L}\right)$ for the transition between states with energies $\epsilon_{i}^{x}, \epsilon_{j}^{x}$. These rates depend on the phonon distribution through $T_{L}$ and can be evaluated from the exciton-acoustical-phonon interaction Hamiltonian. The evolution of the occupation numbers $N_{i}^{x}$ in the exciton reservoir leads to an expression for the rate of change of the total energy:

$$
\begin{aligned}
\left.\frac{d e_{x}}{d t}\right|_{p h}= & \left.\frac{\rho_{x}}{2 \pi} \sum_{i} \Delta E \epsilon_{i}^{x} \frac{d N_{i}^{x}}{d t}\right|_{p h} \\
= & \frac{\rho_{x} \Delta E}{2 \pi} \sum_{i} \epsilon_{i}^{x} \sum_{j}\left[W_{j \rightarrow i}^{p h}\left(T_{L}\right) N_{j}^{x}-W_{i \rightarrow j}^{p h}\left(T_{L}\right) N_{i}^{x}\right] \\
= & \frac{\Delta E n_{x}}{k_{B} T_{x}} \sum_{i} \epsilon_{i}^{x} \sum_{j}\left[W_{j \rightarrow i}^{p h}\left(T_{L}\right) e^{-\epsilon_{j}^{x} / k_{B} T_{x}}\right. \\
& \left.-W_{i \rightarrow j}^{p h}\left(T_{L}\right) e^{-\epsilon_{i}^{x} / k_{B} T_{x}}\right] .
\end{aligned}
$$

For the calculation of $W^{p h}$ we have used the excitonacoustical-phonon coupling through the deformationpotential interaction. These rates have been presented in several previous works, ${ }^{18,26,29}$ and we will not rewrite them here. We consider deformation potentials $a_{e}=2.1 \mathrm{eV}, a_{h}$ $=5.1 \mathrm{eV}$ for $\mathrm{CdTe}^{30}$ and an $80 \AA \mathrm{QW}$. In Eq. (21), stimulated terms are neglected due to the small occupation numbers in the exciton reservoir. The rate of change for $e_{x}$ is linear in $n_{x}$ and dependent on both $T_{L}$ and $T_{x}$.

\section{Cooling to the pump temperature}

The injection of excitons into the reservoir by a pump that is considered to be at the lattice temperature tries to keep the exciton reservoir at $T_{L}$. The terms that correspond to pumping and radiative losses in Eqs. (12),

$$
\left.\frac{d n_{x}}{d t}\right|_{p u}=p_{x}-\Gamma_{x} n_{x},
$$

imply that the rate for the evolution of the occupation numbers has the contribution

$$
\left.\frac{d N_{k}^{x}}{d t}\right|_{p u}=\frac{2 \pi p_{x}}{\rho_{x} k_{B} T_{L}} e^{-\epsilon_{k}^{x} / k_{B} T_{L}}-\Gamma_{x} \frac{2 \pi n_{x}}{\rho_{x} k_{B} T_{x}} e^{-\epsilon_{k}^{x} / k_{B} T_{x}},
$$

where the condition that the pump injects excitons at $T_{L}$ into a Maxwell-Boltzmann distribution at $T_{x}$ is included. The rates in Eq. (23) lead to an additional contribution to the evolution of $e_{x}$

$$
\left.\frac{d e_{x}}{d t}\right|_{p u}=\left.\frac{1}{S} \sum_{k} \epsilon_{k}^{x} \frac{d N_{k}^{x}}{d t}\right|_{p u}=p_{x} k_{B} T_{L}-\frac{n_{x}}{\tau_{x}} k_{B} T_{x} .
$$

Note that this contribution cools the exciton reservoir if $T_{x}>T_{L}$.

The set of first-order differential equations (12), together with Eqs. (20), (21), and (24), leads to a closed description for the dynamics of the exciton reservoir and lower polaritons. We have integrated numerically these equations until the stationary solution $\left(d N^{l p} / d t=d n_{x} / d t=d e_{x} / d t=0\right)$ is 


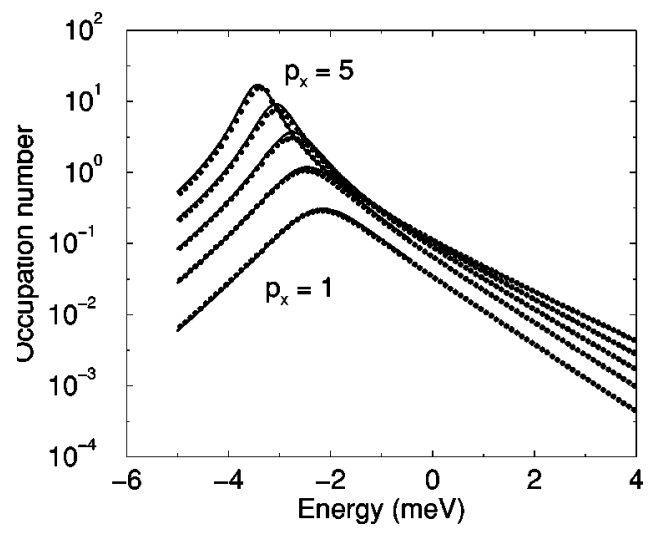

FIG. 2. Polariton distribution calculated by means of the complete semiclassical Boltzmann equation, as in Ref. 18 (points), and polariton distribution calculated by means of our simplified model (continuous line). We have considered a CdTe microcavity with zero detuning and $T_{L}=10 \mathrm{~K}$. The curves, from bottom to top, refer to pump powers from 1 to 5 , in units of $10^{10} \mathrm{~cm}^{-2} / 100 \mathrm{ps}$.

reached. The obtained solution describes the steady state for the case of a continuous non-resonant pump.

\section{Comparison between our simplified model} and the complete semiclassical Boltzmann equation

We have checked whether the polariton distribution obtained with this simplified model agrees with that obtained in a detailed calculation performed with a Boltzmann equation, in which the simplifying assumption of a MaxwellBoltzmann distribution for the levels in the exciton reservoir is not made. In Fig. 2 we present the comparison between a complete calculation that follows Ref. 18, with a grid uniform in energy $(\Delta E=0.1 \mathrm{meV})$, in which all the polaritonpolariton and polariton-phonon scattering processes are included, and our simplified model. We expect that the results from both calculations coincide under conditions for which Bose-Einstein condensation (that is, the macroscopic occupancy of a single microscopic mode) is not possible. On the contrary, in the range of densities near or above BEC, comparison between both models would not be allowed because the system would not be free to relax into a single microscopic mode. Thus, a continuum density of states would not be able to describe properly the LPB, and a grid uniform in energy cannot be applied. In Fig. 2 a few pump powers have been chosen for which the comparison is possible. It can be clearly seen that both calculations yield identical results, so that we can conclude that our simplified model captures the essential physics in the relaxation from the exciton reservoir towards the lower-energy polariton states.

\section{EVOLUTION OF THE POLARITON DISTRIBUTION WITH PUMP POWER AND TEMPERATURE}

\section{A. Evolution with temperature}

In the preceding section, we obtained an estimation for the evolution of the polariton population as the exciton temperature $T_{x}$ is increased. A rough linear relation between the position of the maximum in the distribution and $T_{x}$ was pre-

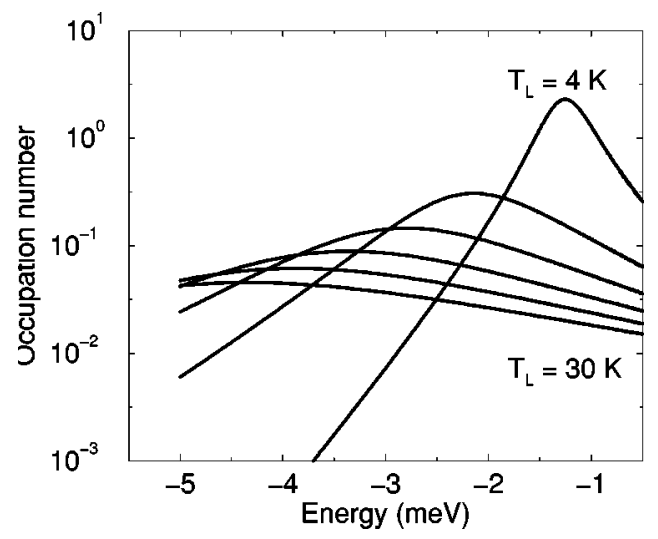

FIG. 3. Evolution of the polariton distribution for $T_{L}$ $=4,10,15, \ldots, 30 \mathrm{~K}, \Omega_{P}=10 \mathrm{meV}$, zero detuning, and $p_{x}$ $=10^{10} \mathrm{~cm}^{-2} / 100 \mathrm{ps}$.

dicted. Now we present a complete calculation with our model, which confirms this estimate. This effect can be observed for a different range of polariton splittings and detunings. However, the main parameter that governs the relaxation from the exciton reservoir is the energy difference between the bare exciton and the bottom of the LPB. This energy distance can be interpreted as the depth of the polariton trap formed by the dip in the polariton dispersion. Within this paper we will consider, for concreteness, the case of zero detuning [that is, $E_{x}$ (bare exciton energy) $=E_{c}$ (bare photon energy)], so that the lowest polariton mode has an energy $\Omega_{P} / 2$ below $E_{X}$.

In Fig. 3 we show the evolution of the polariton distribution for $\Omega_{P}=10 \mathrm{meV}$. We choose $p_{x}=10^{10} \mathrm{~cm}^{-2} / 100 \mathrm{ps}$. Under such excitation conditions, $X-P$ scattering is slower than radiative recombination in the exciton reservoir and $n_{x}$ $\approx \tau_{x} p_{x}$. The heating of the exciton gas is also negligible, so that $T_{x} \approx T_{L}$. The peak in the polariton distribution moves towards lower energies as the temperature is increased. Due to the fact that the rate given in Eq. (14) for scattering into the LPB decreases with $T_{x}$, smaller occupation numbers are predicted for higher temperatures. At high $T_{x}$, the bottleneck is suppressed and the polariton distribution becomes almost flat. The difference between high and low $T_{x}$ was already observed in Ref. 11, where a flat distribution was evidenced for pump powers for which the $X-P$ scattering is the dominant relaxation mechanism.

The evolution of the peak in the polariton distribution is shown in Fig. 4 for a range of polariton splittings. The linear dependence predicted by the estimation in Eq. (18) is clearly observed. For high enough temperatures and a small energy distance between the lowest polariton energy and the exciton reservoir, the maximum in the polariton distribution can reach the bottom of the LPB. However, the occupation numbers are still smaller than 1 , and the situation is not able to produce BEC.

Due to the isotropy of the lower polariton distribution, the emission at a given energy $\epsilon_{\max }^{l p}$ corresponds to emission at a given angle with the growth axis. The movement of $\epsilon_{\max }^{l p}$ towards lower energies implies that the sample emits light preferentially in a ring, at a given angle that decreases with 


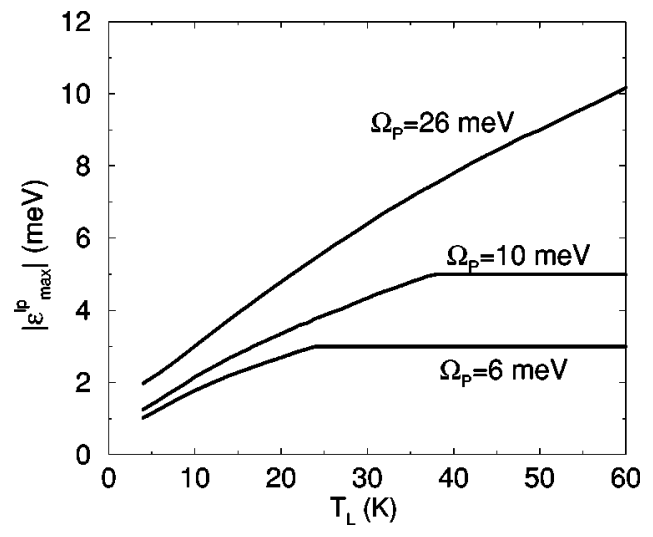

FIG. 4. Dependence of the energy of the peak in the polariton distribution on $T_{L}$ for $p_{x}=10^{10} \mathrm{~cm}^{-2} / 100 \mathrm{ps}$, and different polariton splittings.

temperature. The recent experiment reported by Savvidis et $a l .{ }^{10}$ has observed the ring emission with the linear dependence on lattice temperature described above, and shows that the $X-P$ scattering from a thermalized exciton reservoir considered here is the main scattering mechanism for relaxation into the LPB.

\section{B. Evolution with pump power}

As shown in Eq. (18), $\epsilon_{\max }^{l p}$ depends logarithmically on the exciton density and linearly on $T_{x}$. At large densities, however, the heating of the exciton reservoir must be properly taken into account. When occupation numbers $N_{k}^{l p}>1$ are achieved, the $X-P$ scattering mechanism shown in Fig. 1 is stimulated, and the injection of high-energy excitons becomes more and more important. In this situation, the heating of the exciton reservoir governs the evolution of $\epsilon_{\max }^{l p}$ as $p_{x}$ is increased, because for higher $T_{x}$, the polariton distribution has a maximum at lower energies. This way, the heating of the exciton reservoir allows the excitons to fall into the polariton trap formed by the dip in the polariton dispersion.

Figure 5 shows the evolution of the polariton distribution as one increases the pump power. The distribution can reach

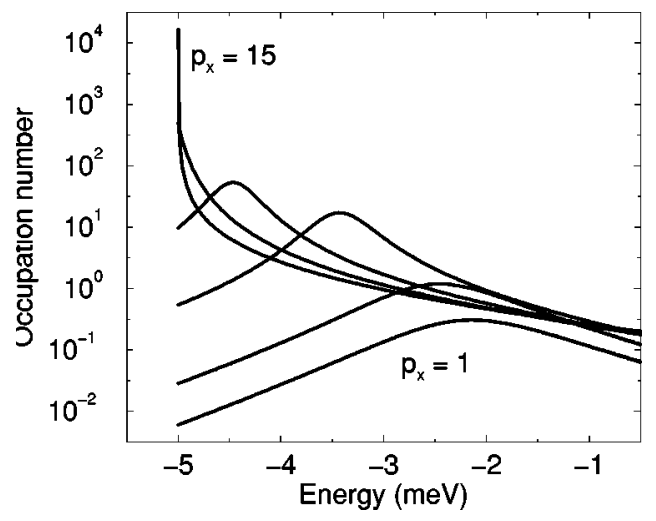

FIG. 5. Evolution of the polariton distribution for $T_{L}=10 \mathrm{~K}$, $\Omega_{P}=10 \mathrm{meV}$, and $p_{x}=1,2,5,8,15 \times 10^{10} \mathrm{~cm}^{-2} / 100$ ps (from bottom to top).

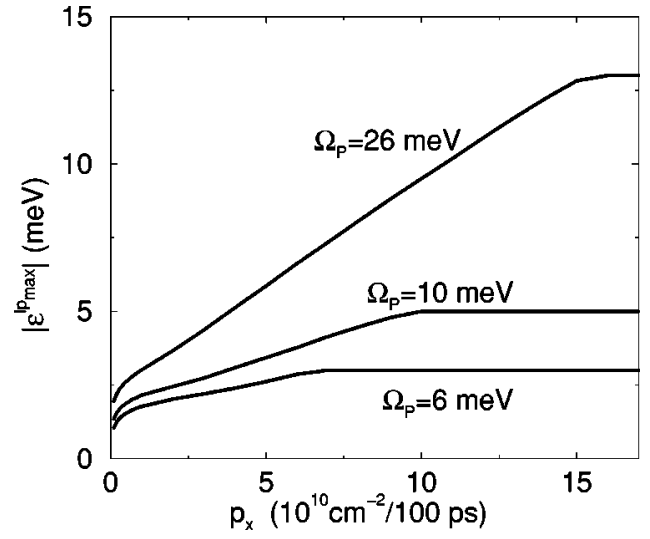

FIG. 6. Dependence of the energy of the peak in the polariton distribution on pump power for $T_{L}=10 \mathrm{~K}$, and different polariton splittings.

the lowest-energy state and large occupation numbers can now be achieved. For large enough powers, the lowestenergy state can have the main part of the polariton population, a situation that will be described in detail in Sec. IV. For intermediate pump powers, occupation numbers larger than 1 are obtained for modes with $k \neq 0$. Thus, our model predicts the stimulation towards these modes before BEC is achieved.

In Fig. 6 we show the evolution of $\epsilon_{\max }^{l p}$ as one increases $p_{x}$ for different $\Omega_{P}$ 's. Three stages can be clearly distinguished. From $p_{x} \approx 10^{9} \mathrm{~cm}^{-2} / 100 \mathrm{ps}$ to $p_{x}$ $\approx 10^{10} \mathrm{~cm}^{-2} / 100 \mathrm{ps}$, the exciton density created by the pump is small enough for the heating of the exciton reservoir to be neglected. Under such conditions, the evolution of $\epsilon_{\max }^{l p}$ is governed by the logarithmic dependence on $n_{x} \approx p_{x} \tau_{x}$ [see Eq. (18)]. Above $p_{x} \approx 10^{10} \mathrm{~cm}^{-2} / 100 \mathrm{ps}$, the heating of the exciton gas is important, and the evolution of $\epsilon_{\max }^{l p}$ is governed by the evolution of $T_{x}$, through the linear dependence shown in Eq. (18). In this regime, $\epsilon_{\max }^{l p}$ depends linearly on $p_{x}$, until it saturates to the bottom of the LPB.

The heating of the exciton reservoir is shown in Fig. 7. For large pump powers, excitons are no longer at the temperature $T_{L}$ at which they are initially injected. The raise of $T_{x}$ becomes more important for larger $\Omega_{P}$ due to the highest energies of the excitons injected into the reservoir by the $X-P$ scattering (see Fig. 1).

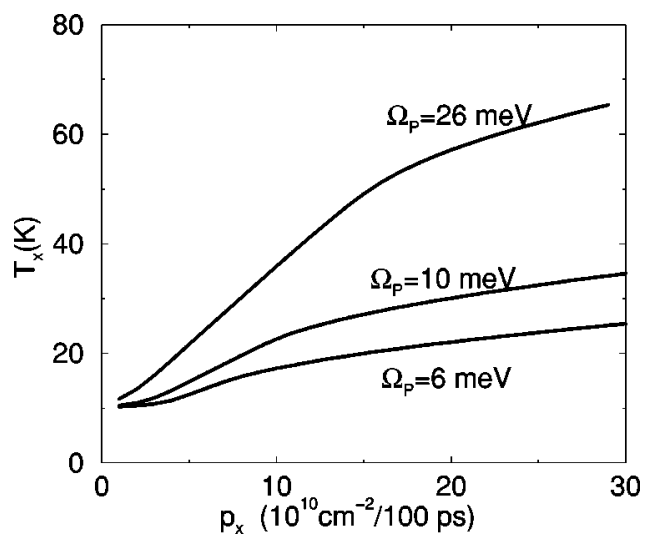

FIG. 7. Dependence of the temperature of the exciton reservoir on pump power for $T_{L}=10 \mathrm{~K}$, and different polariton splittings. 


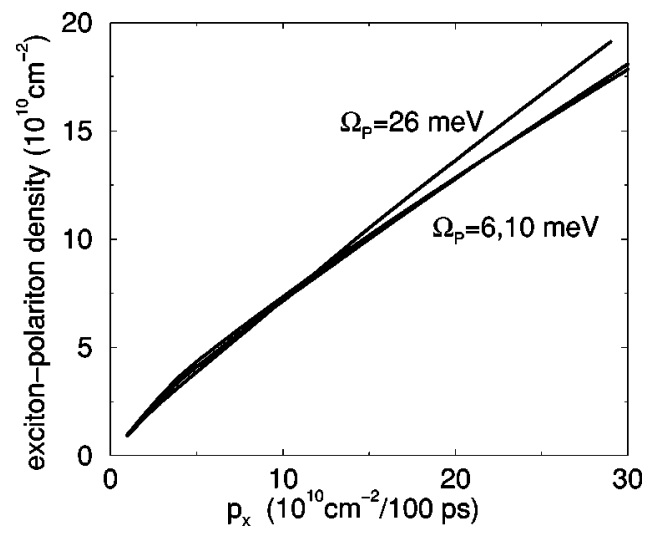

FIG. 8. Dependence of the total exciton reservoir and lower polariton density $n_{x p}$ on pump power for $T_{L}=10 \mathrm{~K}$. The results for $\Omega_{P}=6$ and $10 \mathrm{meV}$ are indistinguishable.

At large $p_{x}, X-P$ scattering is faster than the radiative recombination given by $\tau_{x}$, and it becomes the main mechanism for losses in the exciton reservoir. Thus, the excitonpolariton density $n_{x p}$ (that is, the density of excitons in the reservoir plus the density of lower polaritons) created by the pump is smaller than $p_{x} \tau_{x}$. Figure 8 shows the evolution of the steady-state polariton density as $p_{x}$ is increased. For all the three polariton splittings considered, the evolution of the exciton-polariton density is very similar.

Figure 5 and 8 allow to estimate the $n_{x p}$ at which the relaxation into the ground state is possible. In Fig. 5 pump powers above $5 \times 10^{10} \mathrm{~cm}^{-2} / 100$ ps correspond to polariton densities larger than $4 \times 10^{10} \mathrm{~cm}^{-2}$. Thus in GaAs microcavities, the weak-coupling regime will be reached before $\epsilon_{\max }^{l p}$ reaches the bottom of the LPB dispersion due to the small $n_{\text {sat }}$, and BEC is not possible. For the clean observation of the complete evolution of the polariton distribution towards the ground state, CdTe microcavities are promising candidates because $n_{x p}$ remains well below $n_{\text {sat }}$ before the system relaxes towards the ground state.

The shift of $\epsilon_{\max }^{l p}$ towards lower energies results also in the emission in a ring at an angle that decreases as pump power is increased. In the experiments performed by Savvidis et al., ${ }^{10}$ this effect was observed, and a linear relation between $\epsilon_{\max }^{l p}$ and pump power was reported. In this experiment, the extrapolation of the linear dependence of $\epsilon_{\max }^{l p}$ on $p_{x}$ towards small powers shows an energy offset, in agreement with the theoretical result (see Fig. 6). Angle-resolved measurements allow, thus, to monitor the evolution of the polariton population towards the ground state.

As shown in Fig. 5, for intermediate pump powers the system does not relax down to the ground state, but stimulation (occupancy larger than 1) is achieved in the scattering into intermediate modes with $k \neq 0$. The stimulated modes will thus emit light at angles $\theta \neq 0$. Figure 9 presents the evolution of the occupation numbers for the modes corresponding to different angles as pump power is increased. The evolution of the occupancy at each angle matches the experimental results in Ref. 10.

A quadratic relation is predicted, followed by an exponential growth that saturates at large powers. Stimulation can be

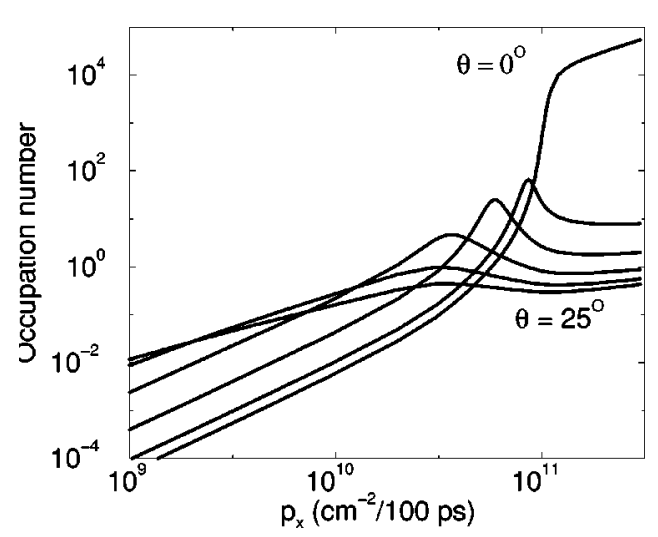

FIG. 9. Evolution of the occupancy of the modes corresponding to emission angles $\theta=0^{\circ}, 5^{\circ} \ldots 25^{\circ}$ (from right to left) as pump power is increased. $T_{L}=10 \mathrm{~K}, \Omega_{P}=10 \mathrm{meV}$.

observed at polariton densities below the BEC at given angles, which correspond to the modes where the polariton distribution peaks, as shown in Fig. 5. The saturation of the emission at a given angle is related to the evolution of the polariton population that "shifts downwards" in energy due to the heating of the exciton reservoir. Thus, the emission at a given angle saturates when the peak in the polariton distribution turns to smaller angles as pump power is increased.

\section{BOSE-EINSTEIN CONDENSATION OF POLARITONS}

In the preceding section it was shown that $X-P$ scattering is efficient enough to create a quasithermal population into the LPB. We will quantify now in detail how the occupancy of the lowest-energy polariton state evolves when the polariton density is increased.

Above a critical pump power, the continuous BoseEinstein distribution shown in Fig. 5 saturates, and the additional excitons added by the pump fall into the bottom of the LPB. Thus, the lower polariton distribution becomes discontinuous and an abrupt increase is achieved in the number of polaritons in the lowest microscopic level, $N_{0}$. Above the threshold for BEC, the macroscopic occupancy of a single microscopic mode opens the possibility for spontaneous symmetry breaking and the appearance of coherence in that mode. The interaction between the Bose-Einstein condensate and the low-energy polariton modes would lead then to the reconstruction of the polariton spectrum as predicted in the well-known Bogoliubov approach. ${ }^{31}$ These effects can modify the relaxation dynamics above threshold, so that our results are not rigorous in this range. However we can predict the critical density and temperature for BEC and the most favorable conditions for its observation.

Figure 10 (left) shows the evolution of $N_{0}$ as a function of $p_{x}$ for different lattice temperatures and polariton splittings. For large $p_{x}$ above the critical pump, the relation between $N_{0}$ and $p_{x}$ becomes linear, and occupation numbers of the order of $5 \times 10^{4}$ polaritons can be reached in the ground-state. In order to interpret the evolution of the ground state occupancy, it is interesting to plot $N_{0}$ [Fig. 10 (right)] as a function of the steady-state exciton-polariton density $n_{x p}$.

The abrupt increase in $N_{0}$ allows us to estimate a critical 


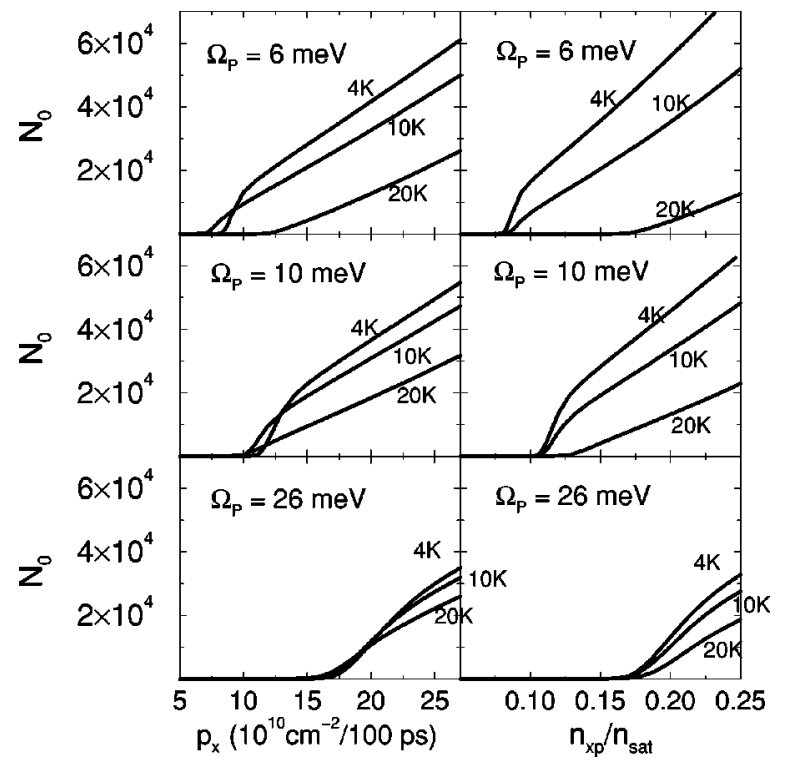

FIG. 10. Left: Evolution of the occupancy of the lowest microscopic polariton level as a function of the pump power. For each polariton splitting we consider $T_{L}=4,10,20 \mathrm{~K}$. Right: Same as above, but now the occupancy of the lowest microscopic level is plotted as a function of the steady-state exciton-polariton density.

density $n_{x p}^{c}$ for BEC, which is $\approx 0.1 n_{s a t}$. However, the situation is now not so clear as in the case of equilibrium BEC, where one expects that $N_{0}$ follows the relation (see Appendix B)

$$
\frac{N_{0}}{S}=n_{x p}-n_{x p}^{c} .
$$

That is, for densities above $n_{x p}^{c}$, all the excitons added to the system should fall into the ground state. Figure 10 (right) shows that for large densities the linear relation is satisfied. However, close to $n_{x p}^{c}, N_{0}$ shows a superlinear dependence on $n_{x p}$. This fact can be interpreted as follows. The equilibrium BEC relation between $N_{0}$ and $n_{x p}$ would be given by the extrapolation of the linear regime of the curves in Fig. 10 (right) towards $N_{0} \approx 0$. By this method we would obtain the equilibrium $n_{x p}^{c}$, which is smaller than the one we get from our complete nonequilibrium calculation. This means that in a real microcavity, $n_{x p}^{c}$ is larger than that predicted by equilibrium BEC. Obviously, this is due to the radiative losses in the LPB and also to the fact that the system has to evolve towards the ground state, following the steps described in the preceding section. Thus, the $X-P$ scattering has to overcome the radiative losses and the exciton reservoir has to be heated, so that relaxation into the ground state becomes efficient enough. The nonequilibrium critical density has to be larger than the equilibrium one for these conditions to be fulfilled.

The transition from superlinear to linear dependence of $N_{0}$ as a function of $p_{x}$ and $n_{x p}$ is reflected in the evolution of the exciton reservoir temperature $T_{x}$. Figure 7 shows that the heating of the exciton gas slows down for the pump power for which the linear growth of $N_{0}$ starts (see Fig. 10, left

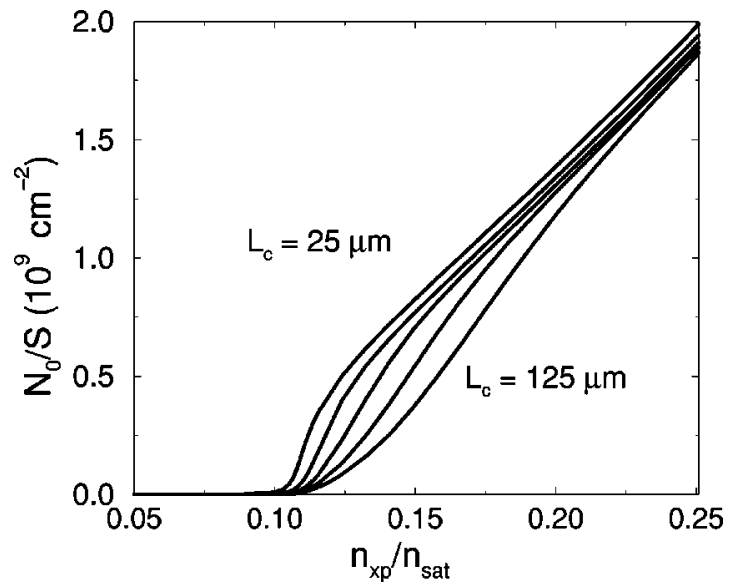

FIG. 11. Evolution of the density of polaritons in the lowest microscopic state, as the exciton-polariton density is increased, for different quantization lengths, $L_{c}=25,50-125 \mu \mathrm{m}$, and $\Omega_{P}$ $=10 \mathrm{meV}, T_{L}=10 \mathrm{~K}$.

panel, $\Omega_{P}=10 \mathrm{meV}$ ). The same behavior in the evolution of $T_{x}$ has been obtained for all the $\Omega_{P}$ 's considered.

We can also see in Fig. 10 that for small polariton splittings, smaller pump powers and polariton densities are needed for BEC, because the energy distance between the bottom of the LPB and the exciton reservoir is also smaller. For large $\Omega_{P}$, the exciton gas reaches higher temperatures in the steady-state regime and the $X-P$ scattering rates are slower (thus, larger polariton densities are needed in order to get BEC). However, in all cases considered in both figures, the critical density is well below $n_{\text {sat }}$.

Up to now we have assumed $L_{c}=50 \mu \mathrm{m}$, of the order of typical spot diameters. However, $L_{c}$ is not a well determined quantity in experiments. Equilibrium BEC in 2D systems predicts that both critical density and temperature depend only on the order of magnitude of $L_{c}$. In order to check what happens in our nonequilibrium situation, we have calculated the density of polaritons in the ground state, $N_{0} / S$, for different quantization lengths (see Fig. 11).

The occupancy of the lowest polariton mode has a more abrupt increase for smaller $L_{c}$. However $n_{x p}^{c}$ is of the order of $0.1 n_{\text {sat }}$ for all the quantization lengths considered.

\section{CONCLUSIONS}

Our main conclusions are the following

(i) At large densities, relaxation after continuous nonresonant excitation can be described by a model in which lower polaritons are injected from a thermalized exciton reservoir by $X-P$ scattering.

(ii) Our model shows a linear relation between the energy of the maximum in the lower polariton distribution and the exciton temperature or pump power. By increasing the exciton density, the heating of the exciton reservoir allows the excitons to overcome the phase-space restrictions and fall into the LPB, which becomes a polariton trap in reciprocal space.

(iii) Bose-Einstein condensation is possible for densities well below $n_{\text {sat }}$ for CdTe microcavities. 
(iv) The most favorable conditions for BEC are small polariton splittings, due to the smaller difference between the bare exciton energy and the bottom of the LPB.

(v) Our results have a weak dependence on the quantization length, which is taken as the excitation spot diameter.

Up to now, nonresonant experiments performed in GaAs microcavities confirm that the weak-coupling regime is reached before $X-P$ scattering allows polaritons to relax towards the ground state. ${ }^{10,12}$ Experimental evidence for the intermediate stage in the evolution of the polariton distribution has been achieved in a GaAs microcavity, ${ }^{10}$ and the experimental results agree very well with the results presented in Sec. III. In CdTe, experimental evidence for stimulation towards the lower polariton states has been achieved under pulsed nonresonant excitation. The angle-resolved measurements after pulsed excitation in CdTe presented in Ref. 32 qualitatively agree with our results for the evolution of the polariton distribution as pump power is increased. However, the physical situation in pulsed experiments is quite different than that considered here, because the system is not allowed to reach the steady state in the relaxation process. Continuous-wave excitation seems to be a cleaner experimental situation for the study of the evolution of the polaritons towards a quasiequilibrium distribution. Our model allows to predict not only the possibility of BEC, but also quantitatively describes the evolution of the system towards the ground state. Angle-resolved measurements under continuous nonresonant excitation would allow to show the macroscopic occupancy of the $k=0$ mode, and also to monitor the evolution towards BEC as pump power is increased.

\section{ACKNOWLEDGMENTS}

We are grateful to Michele Saba, Luis Viña, Francesco Tassone, Ulrich Rössler, Antonio Quattropani, and Paolo Schwendimann for fruitful discussions. D.P. acknowledges the hospitality of the École Polytechnique Fédéral de Lausanne. This work was supported in part by MEC (Spain) under Contract No. N PB96-0085 and CAM (Spain) under Contract No. CAM 07N/0064/2001.

\section{APPENDIX A: CALCULATION OF THE $X$-P SCATTERING RATES}

The calculation of the rates for the scattering of thermalized excitons into the LPB was performed in Ref. 18. Here we reproduce for clarity the main steps and calculate also the rate for ionization out of the LPB.

We consider first the process that injects polaritons. It is given by

$$
\begin{aligned}
W_{k}^{i n} n_{x}^{2}\left(1+N_{k}^{l p}\right)= & \frac{4}{\hbar \pi^{3}}\left|M_{k}\right|^{2} \rho_{x}^{3} \int R\left(\epsilon_{i}^{x}, \epsilon_{j}^{x}, \epsilon_{f}^{x}, \epsilon_{k}^{l p}\right) \\
& \times N_{i}^{x} N_{j}^{x}\left(1+N_{k}^{l p}\right) d \epsilon_{i}^{x} d \epsilon_{j}^{x} .
\end{aligned}
$$

$N_{i}^{x}, N_{j}^{x}$ are the occupation numbers for the initial excitons in the reservoir, and $N_{f}^{x}$ and $N_{k}^{l p}$ correspond to the final exciton and lower polariton, respectively. The $N^{x}$ in the thermalized exciton reservoir follow the relation (8). Due to their large density of sates we can neglect the stimulation terms in the exciton reservoir $\left(N^{x} \ll 1\right)$. Energy conservation is implicit in Eq. (A1), so that $\epsilon_{f}=\epsilon_{i}+\epsilon_{j}-\epsilon_{k}^{l p}$. The function $R$ describes the phase-space restrictions for the problem of excitonexciton scattering in $2 \mathrm{D}$, under the approximation of isotropic exciton distributions. It can be expressed in terms of the corresponding in-plane momenta: ${ }^{18}$

$$
\begin{aligned}
R\left(\epsilon_{i}^{x}, \epsilon_{j}^{x}, \epsilon_{f}^{x}, \epsilon_{k}^{l p}\right)= & \int \frac{1}{2} d x\left\{\left[\left(k_{i}^{x}+k^{l p}\right)^{2}-x\right]\left[x-\left(k_{i}^{x}-k^{l p}\right)^{2}\right]\right. \\
& \left.\times\left[\left(k_{j}^{x}+k_{f}\right)^{2}-x\right]\left[x-\left(k_{j}^{x}-k^{f}\right)^{2}\right]\right\}^{-1 / 2},
\end{aligned}
$$

where integration is limited to the interval where the argument of the square root is positive. A great simplification is obtained when we take the limit $k^{l p} \ll k^{x}$ :

$$
R\left(\epsilon_{i}^{x}, \epsilon_{j}^{x}, \epsilon_{f}^{x}, \epsilon_{k}^{l p}\right)=\frac{\pi}{8 \rho_{x}} \frac{1}{\sqrt{\epsilon_{i}^{x} \epsilon_{j}^{x}-\left(\epsilon_{i}^{l p} / 2\right)^{2}}},
$$

If we insert this expression into Eq. (A1), we obtain

$$
\begin{aligned}
W_{k}^{i n}= & \frac{2 \pi}{\hbar\left(k_{B} T_{x}\right)^{2}}\left|M_{k}\right|^{2} \int \frac{1}{\sqrt{\epsilon_{i}^{x} \epsilon_{j}^{x}-\left(\epsilon_{i}^{l p} / 2\right)^{2}}} \\
& \times e^{-\left(\epsilon_{i}^{x}+\epsilon_{j}^{x}\right) / k_{B} T_{x}} d \epsilon_{i}^{x} d \epsilon_{j}^{x},
\end{aligned}
$$

which after integration in energy gives the expression reported in Eq. (14).

The calculation of the rates for scattering out of the LPB follows the same way:

$$
\begin{aligned}
W_{k}^{\text {out }} n_{x} N_{k}^{l p}= & \frac{4}{\hbar \pi^{3}}\left|M_{k}\right|^{2} \rho_{x}^{3} \\
& \times \int R\left(\epsilon_{f}^{x}, \epsilon_{k}^{l p}, \epsilon_{i}^{x}, \epsilon_{j}^{l p}\right) N_{f}^{x} N_{k}^{l p} d \epsilon_{i}^{x} d \epsilon_{j}^{x} .
\end{aligned}
$$

The factor $R$ is again given by Eq. (A3) because phase-space restrictions are the same as in the previous case,

$$
\begin{aligned}
W_{k}^{\text {out }}= & \frac{\rho_{x}}{\hbar \pi\left(k_{B} T_{x}\right)^{2}}\left|M_{k}\right|^{2} \\
& \times \int \frac{1}{\sqrt{\epsilon_{i}^{x} \epsilon_{j}^{x}-\left(\epsilon_{i}^{l p} / 2\right)^{2}}} e^{-\epsilon_{f}^{x} / k_{B} T_{x}} d \epsilon_{i}^{x} d \epsilon_{j}^{x} .
\end{aligned}
$$

$\epsilon_{f}^{x}$ can be expressed in terms of $\epsilon_{i}^{x}, \epsilon_{j}^{x}$, and the expression in Eq. (14) is obtained.

\section{APPENDIX B: BOSE-EINSTEIN CONDENSATION IN TWO DIMENSIONS}

Let us consider an ensemble of free bosons that follow a Bose-Einstein distribution with chemical potential $\mu<0$ and 
finite temperature $T$. The density of particles can be evaluated as

$$
n(\mu, T)=\frac{1}{2 \pi} \int_{0}^{\infty} \rho(\epsilon) \frac{d \epsilon}{e^{(\epsilon-\mu) / k_{B} T}-1} .
$$

In 3D, $\rho(\epsilon) \propto \sqrt{\epsilon}$, and the continuous Bose-Einstein distribution with $\mu=0$ gives a finite critical density $n_{c}(T)$. For densities $n>n_{c}(T)$, all the extra particles fall into the ground state.

In $2 \mathrm{D}, \rho=m / \hbar^{2}$ (constant), and the integration in Eq. (B1) diverges as $\mu \rightarrow 0$. For an infinite system, BEC is thus not possible at finite temperature. For a finite system, we can introduce a quantization length $L_{c}$, such that the integration in Eq. (B1) has a low-energy cutoff $\epsilon_{c}$ :

$$
n(\mu, T)=\frac{m}{2 \pi \hbar^{2}} \int_{\epsilon_{c}}^{\infty} \frac{d \epsilon}{e^{(\epsilon-\mu) / k_{B} T}-1}
$$

For the case $\epsilon_{c} / k_{B} T \ll 1$, Eq. (B2) gives a very simple result for the maximum density that the $2 \mathrm{D} \mathrm{BE}$ distribution can accommodate:

$$
n_{c}(T)=-\frac{m k_{B} T}{2 \pi \hbar^{2}} \ln \left(\frac{\epsilon_{c}}{k_{B} T}\right) .
$$

Whenever condition $n>n_{c}(T)$ is satisfied, the extra particles added to the system fall into the ground state:

$$
\frac{N_{0}(T)}{S}=n-n_{c}(T) .
$$

For free particles $\epsilon_{c} \propto E_{c}^{-2}$ and Eq. (B2) allows to predict a weak logarithmic dependence of the critical density on the quantization length. This result qualitatively explains the weak dependence of the critical density on $L_{c}$, obtained in the case of nonequilibrium BEC discussed above.
${ }^{1}$ A. Imamoglu, R.J. Ram, S. Pau, and Y. Yamamoto, Phys. Rev. A 53, 4250 (1996).

${ }^{2}$ F. Tassone, C. Piermarocchi, V. Savona, A. Quattropani, and P. Schwendimann, Phys. Rev. B 56, 7554 (1997).

${ }^{3}$ Markus Müller, Joël Bleuse, and Règis Andrè, Phys. Rev. B 62, 16886 (2000).

${ }^{4}$ R. Huang, F. Tassone, and Y. Yamamoto, Phys. Rev. B 61, R7854 (2000).

${ }^{5}$ P.G. Savvidis, J.J. Baumberg, R.M. Stevenson, M.S. Skolnick, D.M. Whittaker, and J.S. Roberts, Phys. Rev. Lett. 84, 1547 (2000).

${ }^{6}$ J.J. Baumberg, P.G. Savvidis, R.M. Stevenson, A.I. Tartakovskii, M.S. Skolnick, D.M. Whittaker, and J.S. Roberts, Phys. Rev. B 62, R16 247 (2000).

${ }^{7}$ C. Ciuti, P. Schwendimann, B. Deveaud, and A. Quattropani, Phys. Rev. B 62, R4825 (2000).

${ }^{8}$ C. Ciuti, P. Schwendimann, and A. Quattropani, Phys. Rev. B 63, 041303 (2001).

${ }^{9}$ D.M. Whittaker, Phys. Rev. B 63, 193305 (2001).

${ }^{10}$ P.G. Savvidis, J.J. Baumberg, D. Porras, D.M. Whittaker, M.S. Skolnick, and J.S. Roberts, Phys. Rev. B 65, 073309 (2002).

${ }^{11}$ A.I. Tartakovskii, M. Emam-Ismail, R.M. Stevenson, M.S. Skolnick, V.N. Astratov, D.M. Whittaker, J.J. Baumberg, and J.S. Roberts, Phys. Rev. B 62, R2283 (2000).

${ }^{12}$ P. Senellart, J. Bloch, B. Sermage, and J.Y. Marzin, Phys. Rev. B 62, R16 263 (2000).

${ }^{13}$ M.D. Martín, L. Viña, J.K. Son, and E.E. Mendez, Solid State Commun. 117, 267 (2001); 119, 259 (2001).

${ }^{14}$ Le Si Dang, D. Heger, R. André, F. Bœuf, and R. Romestain, Phys. Rev. Lett. 81, 3920 (1998).

${ }^{15}$ M. Saba, C. Ciuti, J. Bloch, V. Thierry-Mieg, R. André, Le Si Dang, S. Kundermann, A. Mura, G. Bongiovanni, J.L. Staehli, and B. Deveaud, Nature (London) 414, 731 (2002).

${ }^{16}$ M. Kira, F. Jahnke, S.W. Koch, J.D. Berger, D.V. Wick, T.R. Nelson, Jr., G. Khitrova, and H.M. Gibbs, Phys. Rev. Lett. 79,
5170 (1997).

${ }^{17}$ G. Khitrova, H.M. Gibbs, F. Jahnke, M. Kira, and S.W. Koch, Rev. Mod. Phys. 71, 1591 (1999).

${ }^{18}$ F. Tassone and Y. Yamamoto, Phys. Rev. B 59, 10830 (1999).

${ }^{19} \mathrm{~A}$ similar method has allowed to describe the formation of a Bose-Einstein Condensate in atomic gases. C.W. Gardiner, M.D. Lee, R.J. Ballagh, M.J. Davis, and P. Zoller, Phys. Rev. Lett. 81, 5266 (1998).

${ }^{20}$ J.J. Baumberg, P.G. Savvidis, P.G. Lagoudakis, M.D. Martin, D. Whittaker, R. Butte, M. Skolnick, and J. Roberts, Physica E (Amsterdam) 13, 385 (2002).

${ }^{21}$ G. Malpuech, A. Kavokin, A. Di Carlo, and J.J. Baumberg, Phys. Rev. B 65, 153310 (2002).

${ }^{22}$ S. Schmitt-Rink, D.S. Chemla, and D.A.B. Miller, Phys. Rev. B 32, 6601 (1985).

${ }^{23}$ The term Rabi splitting $\left(\Omega_{R}\right)$ is also used for the polariton splitting in this context. We have changed here the traditional terminology in order to avoid confusion with the case of splitting in a two-level fermionic system, where the term Rabi splitting was initially introduced.

${ }^{24}$ E. Hanamura, J. Phys. Soc. Jpn. 29, 50 (1970).

${ }^{25}$ J.J. Hopfield, Phys. Rev. 112, 1555 (1958).

${ }^{26}$ A.L. Ivanov, P.B. Littlewood, and H. Haug, Phys. Rev. B 59, 5032 (1999).

${ }^{27}$ F. Tassone, C. Piermarocchi, V. Savona, A. Quattropani, and P. Schwendimann, Phys. Rev. B 53, R7642 (1996).

${ }^{28}$ F. Tassone and Y. Yamamoto, Phys. Rev. A 62, 063809 (2000).

${ }^{29}$ C. Piermarocchi, F. Tassone, V. Savona, A. Quattropani, and P. Schwendimann, Phys. Rev. B 55, 1333 (1997).

${ }^{30}$ Su-Huai Wei and Alex Zunger, Phys. Rev. B 60, 5404 (1999).

${ }^{31}$ S.A. Moschkalenko and D.W. Snoke, Bose-Einstein Condensation of Excitons and Biexcitons (Cambridge University Press, Cambridge, 2000).

${ }^{32}$ Markus Müller, PhD thesis, Université Joseph Fourier, Grenoble, 2000. 